\section{Impacto orçamentário da incorporação de medicamentos para tratamento em segunda linha do edema macular diabético no SUS sob a perspectiva da Secretaria de Estado de Saúde de Minas Gerais, Brasil}

\author{
Budget impact of the incorporation of second- \\ line drug treatment for diabetic macular edema \\ in the Brazilian Unified National Health System \\ from the perspective of the Minas Gerais State \\ Health Department, Brazil
}

\section{Impacto presupuestario de la incorporación de} medicamentos para un tratamiento de segunda línea del edema macular diabético en el Sistema Único de Salud, desde la perspectiva de la Secretaría Estatal de Salud de Minas Gerais, Brasil

\section{Resumo}

Os objetivos foram efetuar a análise do impacto orçamentário para a incorporação de segunda linha terapêutica com terapia antiangiogênica de aplicação intravítrea, para tratamento de edema macular diabético, no âmbito do Sistema Único de Saúde (SUS) em Minas Gerais, Brasil, discutindo sua viabilidade à luz do orçamento do estado. A análise do impacto orçamentário com método determinístico, segundo diretriz do Ministério da Saúde. Foram incluidos os pacientes com provável falha ao tratamento de primeira linha, num horizonte temporal de 5 anos para todas as tecnologias avaliadas. Incluíram-se na análise os medicamentos bevacizumabe (uso off-label), ranibizumabe e aflibercepte. As populações foram calculadas tanto por demanda aferida quanto por estimativa epidemiológica. Como análises de sensibilidade efetuaram-se: cenário com difusão de tecnologia mais lenta; cenário com a entrada de bevacizumabe e ranibizumabe biossimilares no mercado; cenário com a desconsideração da inflação no período. O impacto orçamentário incremental, de acordo com as estimativas de demanda aferida e epidemiológica, respectivamente, foi de $R \$$ 69.493.906,95- $R \$ 473.226 .278$, 78 para bevacizumabe; $R \$ 349.319 .965,60-R \$ 2.378 .732 .103,09$ para ranibizumabe e $R \$ 543.867 .485,47-R \$ 3.703 .524 .490,16$ para aflibercepte. Bevacizumabe foi a alternativa financeiramente mais viável em todos os cenários das estimativas e análises de sensibilidade. Estimou-se incremento próximo a 3\%, comparando com o orçamento de 2016 (demanda aferida). Avalia-se que a incorporação é viável dentro do SUS em Minas Gerais, mas sujeita às prioridades da gestão. A discrepância de preços entre produtos de eficácia semelhante é intrigante e tema fértil para estudos futuros.

Judicialização da Saúde; Edema Macular; Economia da Saúde;

Orçamentos; Sistema Único de Saúde
Jans Bastos Izidoro 1,2

Thais Piazza 3

Eli Iola Gurgel Andrade 3

Juliana Alvares-Teodoro 2

doi: 10.1590/0102-311X00145518

\author{
Correspondência \\ J. B. Izidoro \\ Faculdade de Farmácia, Universidade Federal de Minas Gerais. \\ Av. Presidente Antônio Carlos 6627, Campus Pampulha, \\ Belo Horizonte, MG 31270-901, Brasil. \\ jansizidoro@gmail.com \\ 1 Secretaria de Estado de Saúde de Minas Gerais, Belo \\ Horizonte, Brasil. \\ 2 Faculdade de Farmácia, Universidade Federal de Minas \\ Gerais, Belo Horizonte, Brasil. \\ 3 Faculdade de Medicina, Universidade Federal de Minas \\ Gerais, Belo Horizonte, Brasil.
}




\section{Introdução}

No Brasil, a Constituição Federal de 1988 (http://www.planalto.gov.br/ccivil_03/constituicao/cons tituicaocompilado.htm) dispõe que "saúde é direito de todo cidadão e dever do Estado", o que forneceu as bases fundamentais para a construção do Sistema Único de Saúde (SUS). Ainda, por intermédio de sua Seção II, consolida as bases para a execução de ações de assistência terapêutica integral, incluindo a farmacêutica e a formulação da política de medicamentos.

A partir de 2011, o processo de incorporação de novas terapias passou a ser mediado pela Comissão Nacional de Incorporação de Tecnologias no SUS (CONITEC), que realiza avaliações de tecnologias de saúde (ATS) sempre que demandada, visando ao assessoramento ao Ministério da Saúde. Essas ATS subsidiam a incorporação/investimento em novas tecnologias, bem como o desinvestimento em tecnologias obsoletas. Nesse processo são analisadas a existência de evidências científicas de eficácia, efetividade e segurança da tecnologia avaliada em comparação às demais, bem como o impacto orçamentário da incorporação 1. Dentre as avaliações que subsidiam a incorporação de tecnologias, a análise de impacto orçamentário consiste em uma das etapas finais das ATS, e pode ser entendida como a avaliação das consequências financeiras advindas da adoção de uma tecnologia em saúde, dentro de um determinado cenário com recursos limitados 2, auxiliando na tomada de decisão por parte do gestor público 3 .

Embora sejam inegáveis as melhorias que as inovações tecnológicas trazem para a saúde, uma das ameaças à integridade de qualquer sistema de saúde é a incorporação tecnológica excessiva, sem análises de benefícios, custos e de bem-estar coletivo ${ }^{4}$. Soma-se a esse fato a característica mercantil da atualmente denominada "inovação em saúde".

Evidências sugerem que as garantias atuais de proteção de monopólio e propriedade intelectual não contribuem para a inovação médica genuína. Em vez disso, verifica-se o desenvolvimento de medicamentos me-too, os quais apresentam apenas avanços incrementais, existindo principalmente porque segmentos do mercado de saúde são muito atraentes para as empresas farmacêuticas 5,6. Em 2016, apenas 15 dos 96 novos produtos ou novas indicações representaram avanço terapêutico, sendo que mais da metade destes novos tratamentos não ofereceram quaisquer benefícios em comparação com os tratamentos existentes. Em contrapartida, o preço de lançamento de 58 medicamentos com indicação de terapia oncológica no mercado estadunidense aumentou em cerca de $12 \%$ ao ano entre 1995 e 2013, embora sem apresentarem aumento significativo na sobrevida em comparação com os medicamentos mais antigos existentes no mercado 5.

Mesmo para os medicamentos com desfechos de custo-efetividade bem avaliados, a questão do preço da inovação em saúde tem sido um desafio. Um exemplo foi o preço de lançamento do sofosbuvir, embora custo-efetivo para hepatite C, sua incorporação consumiria $20 \%$ dos orçamentos destinados à compra de medicamentos na Itália e Espanha, resultando em impacto orçamentário avaliado como inviável 6. Dessa maneira, observa-se um movimento em vários países europeus e nos Estados Unidos na definição de limiares de reembolso, pagamento, estratégias como negociação, descontos em impostos e congelamento de preços, na tentativa de fazer com que as novas tecnologias em saúde sejam economicamente acessíveis 6,7.

Em meio a essa onda de inovações de elevadíssimo preço (e eficácia muitas vezes discutível) verifica-se um aumento na busca pelo direito individual à saúde nos tribunais. A chamada judicialização da saúde, fenômeno propiciado por vários gatilhos tais como demanda reprimida, ausência de procedimentos e tecnologias no elenco, prática clínica de prescritores discordante com os protocolos vigentes, acesso a ações informadas por representantes da indústria farmacêutica e demandas originadas pela não cobertura de planos privados de assistência à saúde 8 .

Em 2016, o anticorpo monoclonal ranibizumabe (nome comercial Lucentis) era o medicamento com o maior número de ações judiciais no Estado de Minas Gerais (Brasil), em sua maioria visando ao tratamento de retinopatia diabética e edema macular diabético. Além de totalizar, à época, cerca de 2.500 ações cadastradas, foi o terceiro medicamento com o maior valor investido em aquisições para atendimento de ações, totalizando gastos de mais de 12 milhões de reais ao governo estadual naquele ano, de acordo com relatórios gerenciais do Sistema Integrado de Administração de Materiais e Serviços do Estado de Minas Gerais (SIAD-MG) 9. 
Em um contexto no qual a efetividade dos procedimentos de fotocoagulação gira em torno de $67 \%$ no tratamento de edema macular diabético 10 , sendo estes os únicos tratamentos sistematicamente disponíveis no SUS, ressalta-se a importância da avaliação de utilização de antiangiogênicos inibidores do fator de crescimento endotelial vascular - VEGF-A (sigla do inglês, vascular endothelial growth factor $A$ ), tal qual o ranibizumabe. Atualmente, também como alternativa terapêutica, existe o medicamento aflibercepte registrado junto à Agência Nacional de Vigilância Sanitária (Anvisa) para o tratamento de edema macular diabético. Ainda temos o bevacizumabe, registrado para tratamentos oncológicos, entretanto, seu uso off-label em edema macular diabético demonstra ter um papel importante na terapêutica, sendo que ele já é utilizado em larga escala por oftalmologistas dos Estados Unidos e Europa 11, além de ter autorização de utilização em degeneração macular relacionada à idade pela Anvisa no Brasil, doença semelhante ao edema macular diabético.

A efetividade e segurança desses medicamentos foram apontadas em revisões sistemáticas e ensaios clínicos recentes, sendo os mesmos sempre considerados superiores ao tratamento com fotocoagulação a laser, entretanto, com poucas diferenças entre si 12,13,14,15. Quando comparados em estudo econômico, utilizando-se análise do tipo Markov, os antiangiogênicos agrupados foram considerados poupadores de recursos em uma perspectiva de terapia para toda a vida, em relação ao não tratamento e ao tratamento com fotocoagulação a laser em monoterapia 16 . Outros estudos recomendam o uso especificamente de bevacizumabe em pacientes com edema macular diabético, devido às economias de custos substanciais para os pagadores públicos 17 , sendo que foi estimado um valor de 3,5 milhões de dólares por QALY (sigla do inglês: quality-adjusted life year - anos de vida ajustados para qualidade) adicional na troca pelo ranibizumabe 16 .

Quanto ao caso específico dos antiangiogênicos, podemos citar ainda alguns aspectos interessantes que compõem esse cenário, tais como a proteção patentária, a regulação de preços, os acordos comerciais entre indústrias, bem como seu poderio econômico, a organização da regulação sanitária no país e a projeção e interferência do Poder Judiciário nessa relação.

Nesse contexto, o presente estudo tem como objetivo analisar um cenário hipotético de incorporação de segunda linha terapêutica para o tratamento do edema macular diabético, com medicamentos antiangiogênicos no âmbito do SUS de Minas Gerais, discutindo a viabilidade da incorporação à luz do orçamento do estado.

\section{Materiais e métodos}

Foi desenvolvida uma análise de impacto orçamentário, na qual foram analisados os anticorpos anti-VEGF, com aprovação para o uso em edema macular diabético pela Anvisa (aflibercepte e ranibizumabe) e o bevacizumabe para um teórico uso off-label, por pertencer à mesma classe que os medicamentos citados e ter apresentado evidências científicas que suportam esta utilização 11,12,13,14,15,17.

Para a estimativa do impacto orçamentário foi utilizado o método determinístico, sendo seguidas as diretrizes sugeridas pelo Ministério da Saúde 2. Foi adotada a perspectiva da Secretaria de Estado da Saúde de Minas Gerais (órgão gestor do SUS no âmbito do Estado de Minas Gerais), com população de pacientes residentes no estado, portadores de edema macular diabético que apresentaram falha terapêutica ao tratamento de primeira linha com fotocoagulação a laser ou panfotocoagulação a laser, com horizonte temporal de 5 anos. O cenário de referência, correspondente à tecnologia atualmente disponível no SUS para a indicação em questão, foi a realização de segunda fotocoagulação a laser ou panfotocoagulação a laser nos pacientes com falha terapêutica, sem a inclusão de segunda linha de tratamento. Os cenários alternativos envolveram o manejo dos pacientes com a doença, a partir da incorporação das tecnologias em avaliação, correspondendo à inclusão da segunda linha de tratamento com cada um dos três medicamentos antigiogênicos ao tratamento dos pacientes com falha terapêutica aos procedimentos realizados na primeira linha de tratamento.

As fontes de dados usadas envolveram informações nacionais (Instituto Brasileiro de Geografia e Estatística - IBGE; Departamento de Informática do SUS - DATASUS; Sistema de Gerenciamento da Tabela de Procedimentos, Medicamentos e OPM (órteses, próteses e materiais especiais) do SUS - SIGTAP; CONITEC; e Rede Brasileira de Avaliação de Tecnologias em Saúde - REBRATS), estaduais (Fundação João Pinheiro, Portal de Compras do Estado de Minas Gerais; Sistema Integrado de 
Gerenciamento da Assistência Farmacêutica - SIGAF e SIAD-MG) e da literatura científica obtida em pesquisa nos portais SciELO, PubMed, endereços eletrônicos de agências de ATS e do The Royal College of Ophthalmologists do Reino Unido 10.

As estimativas foram feitas inicialmente pelo método epidemiológico, sendo que os dados populacionais para o Estado de Minas Gerais foram obtidos do Censo Demográfico de 2010 (IBGE. http:// www.ibge.gov.br/estadosat/perfil.php?lang=\&sigla=mg), e a ponderação por faixa etária pela Pesquisa de Amostra Domiciliar da Fundação João Pinheiro 18.

A prevalência da doença foi calculada por uma inferência seriada tomando-se como base a prevalência do diabetes na população adulta (considerada de 18-100 anos) que é de 6,4\% segundo a Pesquisa Nacional por Amostra de Domicílios de 2013 (PNAD 2013) e a prevalência de pacientes que evoluem para o edema macular (10\%) 19,20, totalizando uma estimativa de $0,64 \%$ da população geral e 96.833 pacientes potencialmente elegíveis para a fotocoagulação. Considerando que o procedimento fotocoagulação a laser tem eficácia de $67 \%$ 10, obteve-se o total de 31.955 que seriam potencialmente elegíveis para a segunda linha de tratamento, por já estarem em falha com a primeira linha de tratamento.

A curva de difusão da tecnologia foi iniciada em $60 \%$ com adição de $10 \%$ ao ano. A estimativa de distribuição de mercado foi derivada da distribuição em número absoluto das ações judiciais no estado para cada medicamento em relação à estimativa epidemiológica (total teórico de pacientes). A estimativa das cotas de mercado baseada apenas no perfil de judicialização das tecnologias é um fato limitante do estudo, porém, constitui uma alternativa dada a inacessibilidade dos dados de Market Share dos medicamentos no país, além da possível incorporação concomitante de mais de um medicamento não ter sido uma proposta do presente trabalho.

Como análise alternativa e complementar optou-se por também realizar o cálculo pelo método de demanda aferida, visando a considerar os gargalos assistenciais e o subdiagnóstico referente ao edema macular diabético. Nesse segundo cenário, a estimativa foi feita com base nos registros de procedimentos de fotocoagulação a laser e panfotocoagulação a laser para o código H36.0 (retinopatia diabética) da Classificação Internacional de Doenças - 10a revisão (CID-10), obtidos via Sistema de Informações Ambulatoriais do SUS - SIA/SUS, em um contexto no qual a população elegível para a segunda linha de tratamento consistiria apenas dos pacientes que acessaram anteriormente a primeira linha no SUS.

Com relação ao método de demanda aferida, segundo relatório extraído do DATASUS, o Estado de Minas Gerais realizou um total de 99.540 procedimentos autorizados e reembolsados de fotocoagulação a laser e panfotocoagulação no período entre 2010 e 2016, totalizando uma média de 14.220 procedimentos por ano. Considerando-se que esses procedimentos teriam como indicação o tratamento de edema macular diabético e que 33\% destes seriam falhas terapêuticas e deveriam ser aplicações intravítreas, chegou-se ao valor de 4.693 aplicações intravítreas em média, ou seja, num máximo de 4.693 olhos a serem tratados por ano.

Neste estudo, optou-se por usar o reajuste inflacionário anual médio calculado de 7\%, considerando-se o período de 2005 a 2016 de reajustes concedidos pela Câmara de Regulação do Mercado de Medicamentos (CMED), órgão ligado à Anvisa. Como esses reajustes são anuais e no contexto brasileiro ocorrem com periodicidade definida, entendeu-se salutar considerá-los, uma vez que os mesmos podem ter grande influência no preço de medicamentos em um período de horizonte temporal de 5 anos.

Os dados de custos dos medicamentos foram obtidos no SIAD-MG em atas de Registro de Preços formalizadas nos anos de 2016-2017 pela administração pública para os itens em questão. Já as estimativas de custos dos procedimentos que existem no SUS para o tratamento da doença foram obtidas dos dados de reembolso no SIGTAP. A análise do fracionamento das ampolas para atendimento foi considerada, sendo que a proporção do rendimento de cada ampola em doses individualizadas foi retirada da avaliação de tecnologias em saúde realizada pelo Ministério da Saúde 21 e de indicativo do Núcleo de Avaliação de Tecnologias em saúde do Hospital das Clínicas da Universidade Federal de Minas Gerais 22, totalizando 0,1 $\mathrm{mL}$ por dose (0,05 para a aplicação + 0,05mL de volume de segurança).

O custo por fracionamento de cada dose foi estimado em $\mathrm{R} \$ 50,0022$ e somado ao custo da aplicação, sendo que não foi considerado o fracionamento para o aflibercepte dado que o volume extraível na apresentação do produto é de $0,1 \mathrm{~mL}$. 
Com relação a fatores que podem modificar o tamanho da população de interesse ao longo dos anos, dados relativos ao aumento da incidência de diabetes foram derivados da Pesquisa Nacional de Saúde (PNS), que relata um aumento de 25\% na prevalência em 8 anos, logo um aumento médio de $3,125 \%$ ao ano 23 . Os dados de mortalidade foram obtidos via DATASUS, sendo verificada uma mortalidade de 24,8 óbitos por 100 mil habitantes. Como todos os tratamentos considerados não apresentam influência direta sobre os níveis glicêmicos e estão vinculados a desfechos relacionados à prevenção de degeneração da visão, a mortalidade foi considerada idêntica para todos os cenários.

Como análises de sensibilidade foram efetuadas: cenário com difusão de tecnologia mais lenta (iniciando em $40 \%$ e aumentando $20 \%$ ao ano); cenário com a entrada de bevacizumabe e ranibizumabe biossimilares no mercado (assumindo-se o desconto de 35\% relativo aos medicamentos genéricos); e com a desconsideração da inflação no período. A possibilidade da entrada de biossimilares no mercado com o fim da patente dos anticorpos pode alterar profundamente a dinâmica de preços, de forma que a análise em questão é fundamental para atenuar esta limitação.

Por fim, a análise de custos evitados foi incluída também como análise de sensibilidade, uma vez que o tratamento com antiangiogênicos per se não teria influência direta nas taxas de mortalidade ou eventos adversos decorrentes da doença de base, o diabetes. O único evento diretamente evitado pelos tratamentos seria a perda de acuidade visual até a evolução para cegueira funcional. A valoração de custos para esse tipo de evento é medida de maneira indireta (com perda de anos trabalhados ou valoração da perda da qualidade de vida, por exemplo), sendo apresentada na perspectiva da sociedade, não tendo influência direta palpável na projeção de impacto orçamentário. As estimativas da proporção de casos de cegueira funcional evitados, para ranibizumabe, por meio de um modelo matemático, foram obtidas no estudo de Varma et al. ${ }^{24}$, já o custo social da cegueira foi obtido de um estudo inglês 25 e utilizada a conversão de Libra para Real na data de 11 de abril de 2017, totalizando R \$25.304,79 de custo social por caso de cegueira funcional. Os dados em questão foram inseridos nos campos específicos da Planilha Brasileira de Impacto Orçamentário (PBIO) 2 e dimensionados para a duração de um ano por ciclo, visando à obtenção do output de casos evitados e o valor monetário atribuído. Dada a equivalência terapêutica dos medicamentos foi considerado que a proporção de casos de cegueira evitados seria semelhante entre eles.

\section{Resultados}

A descrição da composição dos custos estimados para cada um dos pacotes terapêuticos analisados (Tabela 1) evidenciou que bevacizumabe custa 3,8 vezes menos que o ranibizumabe, e mais de 10 vezes menos que o aflibercepte. Contudo, considerando-se os procedimentos disponibilizados no SUS, o bevacizumabe apresentou custo superior, sendo $25 \%$ a mais que a panfotocoagulação a laser (R\$ 180,00) e 5 vezes mais que a fotocoagulação a laser (R\$ 45,00), segundo seus preços de reembolso. É interessante frisar que se fosse considerado apenas o preço da dose do medicamento, o bevacizumabe seria cerca de 20 vezes mais barato que o ranibizumabe.

Tabela 1

Cálculo dos custos dos medicamentos antiangiogênicos para tratamento da edema macular diabético (em Reais de 2017).

\begin{tabular}{lccccc}
\hline Medicamento & $\begin{array}{c}\text { Preço por } \\
\text { ampola }\end{array}$ & $\begin{array}{c}\text { Preço da } \\
\text { dose }\end{array}$ & $\begin{array}{c}\text { Exame de } \\
\text { OCT }\end{array}$ & $\begin{array}{c}\text { Custo mensal de } \\
\text { aplicação }\end{array}$ & $\begin{array}{c}\text { Custo de } \\
\text { fracionamento * } \\
\text { final }\end{array}$ \\
\hline Ranibizumabe & $2.671,76$ & $1.335,88$ & 67,50 & 80,00 & 50,00 \\
Aflibercepte & $2.146,47$ & $2.146,47$ & 67,50 & 80,00 & 50,00 \\
Bevacizumabe & $1.126,37$ & 28,16 & 67,50 & 80,00 & 50,00 \\
\hline
\end{tabular}

OCT: tomografia de coerência ótica.

Fonte: elaboração dos autores.

* Informação referente ao ano de 2014. 
O impacto orçamentário incremental de cada um dos medicamentos avaliados no período em estudo, por tipo de estimativa de utilização de demanda epidemiológica e demanda aferida, estão dispostos na Tabela 2. Comparativamente, a eventual incorporação de bevacizumabe seria a opção mais econômica tomando-se o impacto orçamentário incremental em relação à panfotocoagulação (cenário de referência). As incorporações de bevacizumabe, ranibizumabe e aflibercepte gerariam um impacto orçamentário incremental para um período de cinco anos de, respectivamente, $\mathrm{R} \$$ 473.226.278,78, R\$ 2.378.732.103,09 e R\$ 3.703.524.490,16, segundo a estimativa epidemiológica, e $\mathrm{R} \$$ 69.493.906,95, $\mathrm{R} \$ 349.319 .965,60$ e $\mathrm{R} \$ 543.867 .485,47$, segundo a estimativa por demanda aferida. Inicialmente, foi verificado o impacto orçamentário incremental, com os valores projetados para o investimento anual na incorporação de cada um dos três medicamentos estudados representados nas Figuras 1 e 2, de acordo com o tipo de estimativa de demanda adotada.

De acordo com os resultados do modelo de impacto orçamentário, a incorporação de qualquer um dos medicamentos geraria a necessidade de incremento ou remanejamento de cotas no orçamento do estado, sendo que o bevacizumabe seria o medicamento com a incorporação mais econômica. Nota-se, inclusive, que o valor a ser investido em 5 anos para o fornecimento de bevacizumabe seria suficiente para menos de 1 ano de atendimento com os outros medicamentos, independentemente da metodologia para a estimativa populacional.

Tabela 2

Impacto orçamentário incremental de cada estratégia avaliada, para o período de 5 anos, por tipo de estimativa de utilização.

\begin{tabular}{lcc}
\hline Medicamento & Demanda epidemiológica & Demanda aferida \\
\hline Bevacizumabe & $\mathrm{R} \$ 473.226 .278,78$ & $\mathrm{R} \$ 69.493 .906,50$ \\
Ranibizumabe & $\mathrm{R} \$ 2.378 .732 .103,09$ & $\mathrm{R} \$ 349.319 .965,60$ \\
Aflibercepte & $\mathrm{R} \$ 3.703 .524 .490,16$ & $\mathrm{R} \$ 543.867 .485,47$
\end{tabular}

Fonte: elaboração dos autores.

\section{Figura 1}

Impacto orçamentário incremental anual - estimativa epidemiológica.

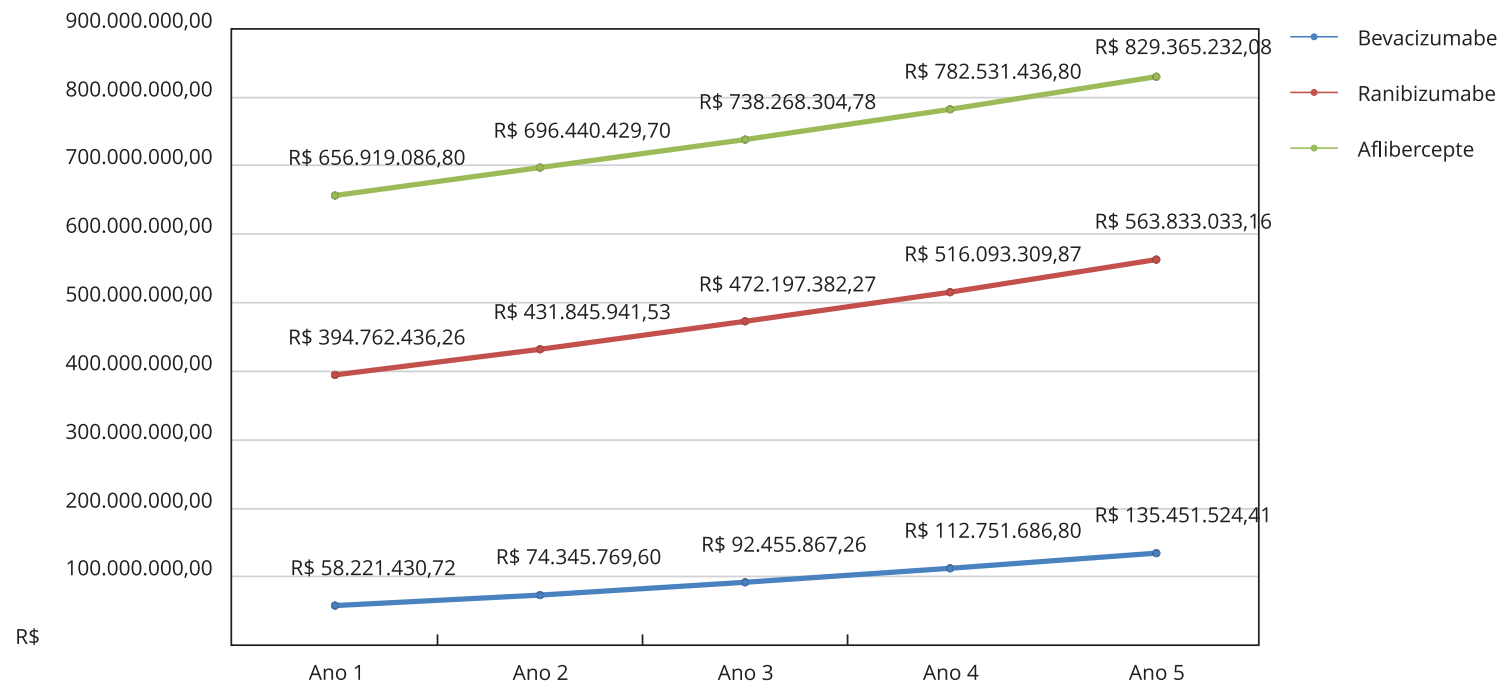




\section{Figura 2}

Impacto orçamentário incremental anual - estimativa por demanda aferida.

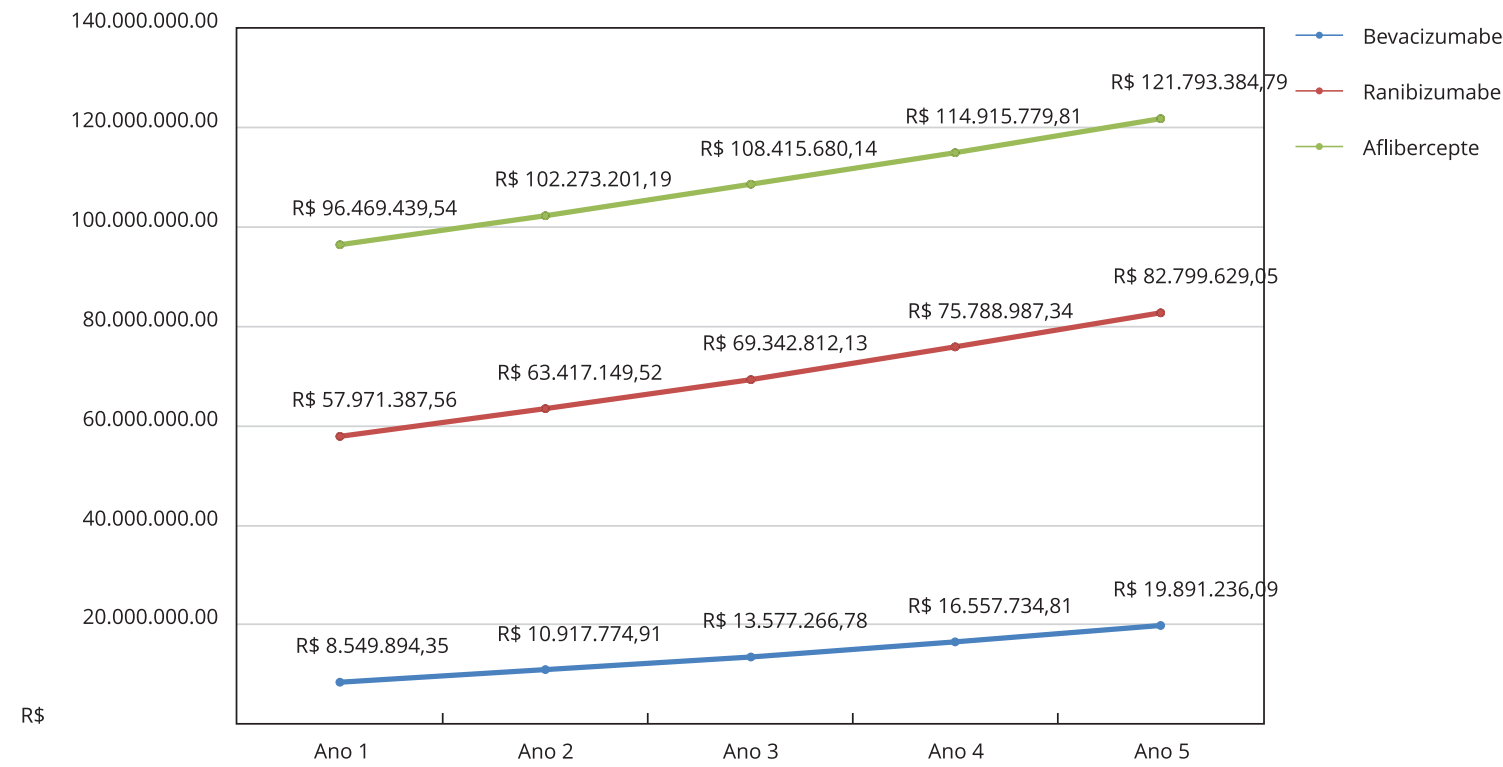

Mesmo sendo mais econômica, a incorporação de bevacizumabe, sob o prisma da demanda aferida, geraria um impacto projetado na faixa de 70 milhões de Reais em um período de 5 anos, o que representaria um gasto médio anual em torno de 14 milhões de Reais.

Nas análises de sensibilidade, para o método da demanda aferida, ao desconsiderarmos a inflação os valores são de R\$ 59.303.305,49, R\$ 302.840.279,26 e R\$ 473.467.421,54. Caso seja considerada uma taxa de difusão mais conservadora e lenta da tecnologia, os valores obtidos são de R\$ 51.862.523,20, R\$ 335.794.744,29 e R\$ 553.984.857,38. Em uma simulação com a inclusão de biossimilar do ranibizumabe (desconto de $35 \%$ ), o valor do impacto do mesmo foi reduzido para R \$ 253.118.837,11, e para bevacizumabe R \$ 51.549.896,19. Por sua vez, em um cenário alternativo no qual aplicou-se todos os requisitos anteriores, os impactos foram de R\$34.160.205,54, R\$ 231.650.417,92 e R\$ 476.244.106,87, respectivamente, como descrito na Tabela 3.

Outra análise de sensibilidade foi desenvolvida com o objetivo de incluir os custos evitados da cegueira funcional a partir do tratamento em segunda linha para edema macular diabético. Com base na análise do referido campo da PBIO, foram adotados a taxa de 69/1.000 casos de cegueira funcional prevenidos ao ano para o ranibizumabe e o custo anual por caso evitado de $\mathrm{R} \$ 25.304,79$, valendose dos estudos norte-americanos e inglês 24,25. Assumiu-se o desempenho equivalente com relação à prevenção de cegueira funcional entre os medicamentos em análise. Nesse cenário, os impactos orçamentários seriam de $\mathrm{R} \$ 57.652 .029,37$ (bevacizumabe), $\mathrm{R} \$ 306.689 .206,34$ (ranibizumabe) e $\mathrm{R} \$ 532.025 .607,90$ (aflibercepte). 
Tabela 3

Análises de sensibilidade do impacto orçamentário de cada estratégia avaliada, para o período de 5 anos, estimativa de demanda aferida.

\begin{tabular}{llll}
\hline Variável & Bevacizumabe & \multicolumn{1}{c}{$\begin{array}{l}\text { Medicamento } \\
\text { Ranibizumabe }\end{array}$} \\
\hline Sem ajuste econômico & $\mathrm{R} \$ 59.303 .305,49$ & $\mathrm{R} \$ 302.840 .279,26$ \\
Difusão conservadora * & $\mathrm{R} \$ 51.862 .523,20$ & $\mathrm{R} \$ 335.794 .744,29$ \\
Entrada de biossimilar ** & $\mathrm{R} \$ 51.549 .896,19$ & $\mathrm{R} \$ 253.118 .837,11$ & $\mathrm{R} \$ 553.984 .857,38$ \\
Todos os requisitos anteriores & $\mathrm{R} \$ 34.160 .205,54$ & $\mathrm{R} \$ 231.650 .417,92$ \\
\hline
\end{tabular}

NA: não se aplica.

Fonte: elaboração dos autores.

* Difusão da tecnologia considerando taxas de 20, 40, 60, 80 e 100\% ao ano;

** Simulação da inclusão de biossimilar do ranibizumabe e bevacizumabe (desconto de 35\%).

\section{Discussão}

Tomando-se como base de análise os valores do orçamento executado em 2016, no Estado de Minas Gerais, é possível traçar alguns panoramas e comparações. Do total de valores liquidados em 2016, 5,37 bilhões de reais foram destinados ao fundo estadual de saúde, ou seja, 6,4\% do orçamento total do estado. Valendo-se do orçamento total liquidado da saúde no período, a incorporação do Bevacizumabe (como incorporação mais econômica) em estudo, a mesma representaria um acréscimo médio de 0,26 e 1,75\% no período de 5 anos estudado, respectivamente, entre as estimativas de demanda aferida e epidemiológica.

Entretanto, o orçamento da saúde é composto de uma variedade de gastos, categorizados por grupos e elementos de despesas. A aquisição de medicamentos está incluída nos elementos de despesas "material de consumo" e "material, bem ou serviço para distribuição gratuita", que totalizam em valores empenhados $\mathrm{R} \$ 534.460 .387,96$, ou seja, cerca de 7,9\% do orçamento projetado da saúde para o ano de 2016. Vale a pena frisar que o valor indicado representa apenas a fração movimentada diretamente pelo fundo estadual de saúde, que seria o financiador natural de um eventual protocolo estadual. Dentro desse universo financeiro, a incorporação de bevacizumabe requereria um acréscimo de 2,6-17,7\% no orçamento destinado à aquisição de medicamentos diretamente pelo Fundo Estadual de Saúde.

Para efeitos de comparação com outros países, verifica-se que o porcentual da estimativa por demanda aferida é semelhante ao que seria praticado no Medicare (Estados Unidos) com o reembolso de aplicações de bevacizumabe aos americanos que o utilizam. Segundo estimativas do National Institute of Health (Estados Unidos), se todos os pacientes utilizassem bevacizumabe ocorreria o consumo de $2 \%$ dos recursos alocados para o bloco B de financiamento (no qual está alocada a oftalmologia). Caso todos os pacientes utilizassem ranibizumabe este consumo seria de $40 \%$, totalizando a relação de 1,7 bilhão de Dólares em 10 anos para bevacizumabe e de 57 bilhões para ranibizumabe, contando com os pacientes que realizam copagamento de $20 \%$, que representam 420 milhões e 14 bilhões de dólares, respectivamente 26 .

Por exemplo, estima-se que a adoção do tratamento utilizando ranibizumabe em detrimento do bevacizumabe na Holanda aumentaria os custos envolvidos em torno de 10 a 15 milhões de Euros, enquanto que no Canadá estima-se que a adoção de ranibizumabe ou aflibercepte, em detrimento do bevacizumabe, geraria um custo para dois anos de tratamento superior em 18.160,00 e 16.171,00 de Dólares por paciente, respectivamente 11,17. Em um estudo com a perspectiva do SUS no nível de gestão federal encontrou-se, inclusive, cenários nos quais a incorporação de bevacizumabe se mostrava poupadora de recursos em um horizonte de 4 anos 27 .

A estimativa pela demanda aferida, nesse sentido, representa a população que efetivamente acessa o sistema, sendo que, devido às limitações da base de dados, ainda existe uma população superestimada, uma vez que o único dado disponível é a quantidade de procedimentos realizados, mas não há a 
quantidade de pacientes atendidos, de forma que houve superestimação do número de olhos a se tratar, pois um olho pode ter sido tratado mais de uma vez num espaço de tempo. Por outro lado, também existe a ocorrência de subdiagnóstico (referente tanto ao diabetes quanto ao edema macular diabético) e de gargalos assistenciais, tornando a estimativa por demanda aferida mais próxima dos pacientes que efetivamente acessariam o sistema. A mensuração desses fatores é complicada, constituindo outra limitação do estudo. Apenas com a finalidade ilustrativa, considerando o tratamento com laser, verificou-se que o mesmo era executado efetivamente apenas em 36 dos 349 serviços tecnicamente habilitados no estado, com aproximadamente $75 \%$ dos procedimentos sendo realizados em apenas quatro prestadores, sendo três de Belo Horizonte e um de Juiz de Fora, Minas Gerais.

Os valores encontrados após as análises de sensibilidade das estimativas de demanda aferida não alteraram substancialmente o ranqueamento entres medicamentos, mas apenas valores absolutos e a relação proporcional entre impactos orçamentários incrementais calculados. Por exemplo, na análise com a taxa de difusão mais lenta, a diferença proporcional entre os impactos orçamentários de bevacizumabe e ranibizumabe passa a ser de aproximadamente 6,5 vezes, tornando a incorporação do bevacizumabe ainda mais atraente neste cenário. Uma conjunção positiva de todos os fatores relacionados à análise de sensibilidade geraria um decréscimo de aproximadamente $50 \%$ no impacto estimado para bevacizumabe, sendo que as outras intervenções isoladamente gerariam economias da ordem de 15\%-25\%. O único fator ajustável pelo gestor dentre os analisados seria a taxa de difusão da tecnologia, sendo que com a situação de escassez de centros de oftalmologia no estado a mesma é um ponto interessante a ser considerado.

A inclusão no modelo de eventos evitados como análise de sensibilidade aconteceu partindo da prerrogativa de que quando um paciente evolui para o estado de cegueira funcional, em tese, ele não geraria um aumento de custos diretos significativo, por se tratar de condição perene e irreversível, inclusive tradicionalmente levando à reclusão e a uma maior chance de diminuir a utilização do sistema de saúde por estes indivíduos. Como em tese o modelo de planilhas determinísticas lida apenas com os custos diretos, somado à escassez e inacessibilidade de dados nacionais de custos e valoração para o desfecho, optou-se pela conversão de dados internacionais dos custos indiretos ou sociais, entretanto, como análise de sensibilidade e não no modelo principal, dada a possível fragilidade dos valores envolvidos.

Naturalmente, a análise de impacto orçamentário não é o único instrumento com o qual o gestor trabalharia para decidir com relação a uma eventual incorporação de tratamento e, consequente, alteração da política pública.

Inúmeros outros fatores influenciam a elaboração de uma politica pública, tais como o arcabouço ideológico de determinado grupo que eventualmente ascender ao poder, o momento financeiro do pagador, as carências assistenciais identificadas concomitantemente, bem como o arranjo dos atores sociais envolvidos, tais como associações de pacientes, mídia, academia, governo, indústria, Poder Judiciário, sendo que tal balanço entre as forças culmina no estabelecimento de uma agenda de prioridades a ser ratificada pelo gestor e pela sociedade 28 .

O caso em estudo reforça, portanto, a tese de que o sistema de concessão de patentes atual, particularmente na área da inovação farmacêutica, está fora de equilíbrio, fornecendo recompensas financeiras excessivas aos detentores patentários, sobretudo das grandes empresas farmacêuticas. No Brasil, essa situação parece ser amplificada, com o preço médio de medicamentos sendo 1,9 vez o praticado na Suécia e 13,1 vezes o índice mundial 29.

Um exemplo está na patente do ranibizumabe, em que a molécula foi desenvolvida pela empresa americana Genentech Inc., a partir da fragmentação do bevacizumabe, apresentando, inclusive, o mesmo número de patente (PI98093879). Embora apresente comparativamente preço mais baixo, verifica-se que a Genentech Inc. desestimula qualquer incentivo à aprovação do bevacizumabe para uso oftalmológico e desencoraja sua prescrição, apesar de toda a evidência científica disponível. A proteção patentária dos itens gera uma reserva de mercado perversa, na qual há dois medicamentos com eficácia semelhante e preços distintos, em que o de maior custo é o mais difundido na prática médica 26.

Tal prática, aparentemente, é executada mundialmente, tanto que existe um acórdão judicial europeu, proveniente de processo originado na Itália, no qual a Autoridade de Garantia da Concorrência e do Mercado (AGCM) aplicou multa à Roche e à Novartis no valor de 90,6 e 92 milhões de Euros, 
respectivamente. A respectiva multa foi justificada pelo fato de que as duas empresas celebraram acordo relativo ao período entre 1o de junho de 2011 e 27 de fevereiro de 2014, que "visava obter uma diferenciação artificial entre os medicamentos Avastin (bevacizumabe) e Lucentis (ranibizumabe), manipulando a percepção dos riscos da utilização do Avastin em oftalmologia” 30.

Dessa forma, o mercado encontra utilidade nos governos como compradores e distribuidores de medicamentos, por intermédio de comunidades específicas mobilizadas. Essas comunidades, consideradas segmentos de mercado terapêutico, usam a legislação e a jurisprudência para terem visibilidade junto ao Estado e para fazê-lo atuar de maneira biopolítica 31.

Em um mundo extremamente desigual, onde empresas muitas vezes têm faturamentos maiores que o PIB de um país em desenvolvimento, elaborar políticas públicas com instrumentos regulatórios para impedir que tais empresas capturem a política é uma tarefa hercúlea. Não são raros os casos de captura de políticas públicas por entes privados, como também são várias as formas em que tal captura acontece 32 .

Há, ainda, problemas inerentes ao processo de regulação sanitária no Brasil, que depende da solicitação das empresas para registrar seus produtos no país. Apesar de ter vantagem competitiva de preços em relação às concorrentes, a empresa detentora da patente do bevacizumabe não demonstra interesse em registrar o produto no país para o tratamento do edema macular diabético. Por outro lado, salta aos olhos o grande número de ações judiciais direcionadas apenas para ranibizumabe, medicamento mais caro, o que acaba exercendo pressão sobre o gestor para a incorporação deste item. No entanto, caso essa incorporação seja realizada, o impacto orçamentário adicional em cinco anos seria de R\$279.823.058,65, somente para o Estado de Minas Gerais, que abriga cerca de 20 milhões de pessoas 18 , o que corresponde a cerca de $10 \%$ da população brasileira se considerarmos o Censo Demográfico de 2010 (Características gerais da população. Resultados da amostra. https://censo2010. ibge.gov.br/).

Para o caso em tela, percebemos que além de um acordo comercial virtualmente espúrio, ainda envolveu-se o Poder Judiciário de modo a se modular uma política pública não orientada apenas pelas necessidades de saúde da população. O fenômeno verificado ilustra uma modalidade mais sutil de captura de políticas/recursos públicos na medida em que envolve o judiciário como modulador e expõe uma faceta perversa das relações econômicas na área da saúde. Utiliza-se do arcabouço legal e do mercado em suas leis e tratados, visando à obtenção de lucro por parte das farmacêuticas, de maneira cada vez mais distanciada da eficiência nos gastos em saúde pública, a despeito dos direitos sociais a uma assistência integral e de qualidade garantida na Constituição.

As perspectivas de se atenuar o problema da pressão de mercado dentro do SUS, ilustradas pelo caso aqui estudado, apresentam prognóstico complicado. Modificações no sentido da maior conscientização do Poder Judiciário quanto ao seu protagonismo na dinâmica da movimentação de recursos públicos, assim como a adoção de políticas apoiadas em evidências, sua divulgação e compreensão pela sociedade e o respeito pelas decisões técnicas em relação às políticas de saúde, tornam-se necessárias. Do mesmo modo que é demandado financiamento adequado do sistema de saúde visando sua autonomia. Um maior aporte financeiro, aliado a uma gestão pautada na eficiência e na saúde baseada em evidências é crucial para que o sistema tenha reconhecimento social e poder de negociação, para amenizar a influência mercadológica e da judicialização na orientação de suas ações. Vale mencionar que uma eventual reformulação das políticas de regulação de preços e de registro de medicamentos aumenta o potencial de dirimir o desperdício de recursos públicos por meio da captura dos mesmos, ao se pagar excessivos dividendos às companhias farmacêuticas.

Em suma, o desafio perpassa pelo empoderamento da saúde pública e de sua organização institucional que é o SUS, visando à instauração de um sistema de saúde público forte e socialmente referendado, no qual haja a confiança dos mais variados atores sociais. 


\section{Colaboradores}

J. B. Izidoro contribuiu na concepção e projeto, análise e interpretação dos dados, redação do artigo. T. Piazza colaborou na redação do artigo, revisão crítica relevante do conteúdo intelectual. E. I. G. Andrade contribuiu na revisão crítica relevante do conteúdo intelectual, aprovação final da versão a ser publicada. J. Alvares-Teodoro colaborou na concepção e projeto, revisão crítica relevante do conteúdo intelectual, aprovação final da versão a ser publicada.

\section{Informações adicionais}

ORCID: Jans Bastos Izidoro (0000-0002-86252676); Thais Piazza (0000-0001-9385-7609); Eli Iola Gurgel Andrade (0000-0002-0206-2462); Juliana Alvares-Teodoro (0000-0002-0210-0721).

\section{Agradecimentos}

Os autores agradecem à Fundação de Amparo à Pesquisa do Estado de Minas Gerais pelo financiamento no projeto por meio do Edital FAPEMIG 14/2013 - Programa de pesquisa para o SUS-PPSUS MS/CNPq/FAPEMIG/SES-Eixo 2.

\section{Referências}

1. Ministério da Saúde. Diretrizes metodológicas. Avaliação de desempenho de tecnologias em saúde. Desinvestimento e reinvestimento. http://conitec.gov.br/images/Consultas/ 2016/diretrizf_investimento_reinvestimento. pdf (acessado em 07/Mar/2019).

2. Ministério da Saúde. Análise de impacto orçamentário manual para o sistema de saúde do Brasil. http://bvsms.saude.gov.br/bvs/publi cacoes/diretrizes_metodologicas_analise_im pacto.pdf (acessado em 31/Jul/2016).

3. Faleiros DR, Álvares J, Almeida AM, Araújo VE, Andrade EIG, Godman BB, et al. Budget impact analysis of medicines: updated systematic review and implications. Expert Rev Pharmacoecon Outcomes Res 2016; 16:257-66.

4. Andrade EIG, Machado CD, Faleiros DR, Szuster DAC, Guerra Jr. AA, Silva GD, et al. A judicialização da saúde e a política nacional de assistência farmacêutica no Brasil: gestão da clínica e medicalização da justiça. Rev Méd Minas Gerais 2008; 18(4 Suppl 4):S46-50.

5. New products and new indications in 2016: a system that favours imitation. Prescrire International 2017; 26:136-9.

6. Flume M, Bardou M, Capri S, Sola-Morales O, Cunningham D, Levin L. Approaches to manage 'affordability' of high budget impact medicines in key EU countries. J Mark Access Health Policy 2018; 6:1478539.

7. Schaffer SK, Messner D, Mestre-Ferrandiz J, Tambor E, Towse A. Paying for cures: perspectives on solutions to the "affordability issue". Value Health 2018; 21:276-9.

8. Osorio-de-Castro CGS, Luiza VL, Castilho SR, Oliveira MA, Jaramillo NM. Assitência farmacêutica gestão e prática para profissionais da saúde. Rio de Janeiro: Editora Fiocruz; 2014.

9. Sistema Integrado de Administração de Materiais e Serviços do Estado de Minas Gerais. Versão 1.08. Sabratec Technologies Inc. https:// www.siad.mg.gov.br/siad/login.jsp (acessado em 07/Mar/2019).

10. Ghanchi F, Bailey C, Chakravarthy U, Cohen S, Dobson P, Gibson J, et al. The Royal College of Ophthalmologists Diabetic Retinopathy Guidelines. Diabetic retinopathy guidelines, 2012. http://www.icoph.org/dynamic/ attachments/taskforce_documents/2012sci-267_diabetic_retinopathy_guidelines_de cember_2012.pdf (acessado em 05/Jul/2017).

11. Schauwvlieghe AME, Dijkman G, Hooymans JM, Verbraak FD, Hoyng CB, Dijkgraaf MGW, et al. Comparing the effectiveness and costs of Bevacizumab to Ranibizumab in patients with Diabetic macular edema: a randomized clinical trial (the BRDME study). BMC Ophthalmol 2015; 15:71.

12. Ford J, Lois N, Royle P, Clar C, Shyangdan D, Waugh N. Current treatments in diabetic macular oedema: systematic review and metaanalysis. BMJ Open 2013; 3:e002269. 
13. Virgili G, Parravano M, Menchini F, Evans J. Anti-vascular endothelial growth factor for diabetic macular oedema. Cochrane Database Syst Rev 2014; (10):CD007419.

14. Wells JA, Glassman AR, Ayala AR, Jampol LM, Aiello LP, Antoszyk AN, et al. Aflibercept, bevacizumab, or ranibizumab for diabetic macular edema. N Engl J Med 2015; 372:1193-203.

15. Régnier S, Malcolm W, Allen F, Wright J, Bezlyak V. Efficacy of anti-VEGF and laser photocoagulation in the treatment of visual impairment due to diabetic macular edema: a systematic review and network meta-analysis. PLoS One 2014; 9:e102309.

16. Pershing S, Enns EA, Matesic B, Owens DK, Goldhaber-Fiebert JD. Cost-effectiveness of treatment of diabetic macular edema. Ann Intern Med 2014; 160:18-29.

17. Deonandan R, Jones S. Anti-vascular endothelial growth factor drugs for the treatment of retinal conditions. Ottawa: Canadian Agency for Drugs and Technologies in Health; 2017.

18. Fundação João Pinheiro. Boletim da Pesquisa por Amostra de Domicílios: indicadores de despesas, 2013. Belo Horizonte: Fundação João Pinheiro; 2014.

19. Sukha AY, Rubin A. Definition, classification and visual aspects of diabetes mellitus, diabetic retinopathy and diabetic macular edema: a review of literature. South African Optometrist 2007; 66:120-31.

20. Comissão Nacional de Incorporação de Tecnologias no SUS. Ranibizumabe para degeneração macular relacionada à idade. Brasília: Ministério da Saúde; 2015. (Relatório de Recomendação, 119).

21. Comissão Nacional de Incorporação de Tecnologias no SUS. Antiangiogênicos (bevacizumbe e ranibizumabe) no tratamento do edema macular diabético. Brasília: Ministério da Saúde; 2015.

22. Núcleo de Avaliação de Tecnologias em Saúde, Hospital das Clínicas, Universidade Federal de Minas Gerais. Ranibizumabe (Lucentis $\left.{ }^{\circledR}\right)$ para tratamento da retinopatia diabética. Resposta técnica 403/2014. http:// www.cnj.jus.br/files/conteudo/destaques/ arquivo/2015/04/2e359513a11bab9e1dbc 8a93671729d1.pdf (acessado em 05/Jul/2017).

23. Iser BPM, Stopa SR, Chueiri PS, Szwarcwald CL, Malta DC, Monteiro HOC, et al. Prevalência de diabetes autorreferido no Brasil: resultados da Pesquisa Nacional de Saúde 2013. Epidemiol Serv Saúde 2015; 24:305-14.
24. Varma R, Bressler NM, Doan QV, Danese M, Dolan CM, Lee A, et al. Visual impairment and blindness avoided with ranibizumab in hispanic and non-hispanic whites with diabetic macular edema in the United States. Ophthalmology 2015; 122:982-9.

25. Meads C, Hyde C. What is the cost of blindness? Br J Ophthalmol 2003; 87:1201-4.

26. Hutton D, Newman-Casey PA, Tavag M, Zacks D, Stein J. Switching to less expensive blindness drug could save medicare part B $\$ 18$ billion over a ten-year period. Health Aff (Millwood) 2014; 33:931-9.

27. Tavares F, Elias S, Nunes Da Silva E, Belfort R, Silva MT, Atallah AN. Treatment options for age-related macular degeneration: a budget impact analysis from the perspective of the Brazilian Public Health System. PLoS One 2015; 10:e0139556.

28. Guerra Jr. AA, Acurcio FA. Política de medicamentos e assistência farmacêutica. In: Acurcio FA, organizador. Medicamentos e assistência farmacêutica. Belo Horizonte: Editora Coopmed; 2003. p. 115-7.

29. Nóbrega ODT, Marques AR, de Araújo ACG, Karnikowski MGDO, Naves JDOS, Silver LD. Retail prices of essential drugs in Brazil: an international comparison. Rev Panam Salud Pública 2007; 22:118-23.

30. InfoCuria. Case-law of the Court of Justice. Case C-179/16. http://curia.europa.eu/juris/ document/document.jsf?docid $=198644 \& \bmod$ $\mathrm{e}=$ req \& pageIndex $=1 \& \operatorname{dir}=\&$ occ $=$ first $\&$ part $=1$ $\&$ text $=\&$ doclang $=$ PT\&cid $=289123$ (acessado em 29/Jan/2018).

31. Biehl J, Petryna A. Tratamentos jurídicos: os mercados terapêuticos e a judicialização do direito à saúde. Hist Ciênc Saúde Manguinhos 2016; 23:173-92.

32. Oliveira Junior HA, Villardi P, Vieira MF, Fonseca F. Produção local de medicamentos e captura coorporativa: análise do caso brasileiro. http://deolhonaspatentes.org/wp-content/ uploads/2016/07/livro_PT_rev1.pdf (acessado em $05 / \mathrm{Jul} / 2017$ ). 


\section{Abstract}

The study's objective was to perform budget impact assessment for the incorporation of secondline intravitreal antiangiogenic therapy for diabatic macular edema in the scope of the Brazilian Unified National Health System (SUS) in Minas Gerais state, Brazil, discussing the incorporation's state budget feasibility. The budget impact assessment was performed as a deterministic method according to Ministry of Health guidelines. The study included patients with probable first-line treatment failure in a five-year timeline for all the technologies assessed. The analysis included the drugs bevacizumab (off-label use), ranibizumab, and aflibercept. The populations were calculated both by observed demand and epidemiological estimate. The following sensitivity analyses were performed: a scenario with slower technology diffusion, a scenario with the market entry of biosimilar versions of bevacizumab and ranibizumab, and a scenario disregarding inflation during the period. The incremental budget impacts according to observed and epidemiologically estimated demand, respectively, were BRL 69,493,906.95 to BRL 473,226,278.78 for bevacizumab; BRL 349,319,965.60 to BRL 2,378,732,103.09 for ranibizumab; and BRL 543,867,485.47 to $B R L$ 3,703,524,490.16 for aflibercept. Bevacizumab proved to be the most financially feasible alternative in all the scenarios of estimates and sensitivity analyses. An increment of nearly 3\% was estimated, compared to the 2016 budget (observed demand). The study showed that the incorporation is feasible in the SUS, Minas Gerais State, but subject to management priorities. Price discrepancies between products with similar efficacy is intriguing and provides fertile ground for future studies.

Health's Judicialization; Macular Edema; Health Economics; Budgets; Unified Health System

\section{Resumen}

El objetivo fue efectuar un análisis del impacto presupuestario en la incorporación de una segunda línea terapéutica, con terapia antiangiogénica de aplicación intravítrea, para el tratamiento de edema macular diabético, en el ámbito del Sistema Unico de Salud (SUS), en Minas Gerais, Brasil, discutiendo su viabilidad respecto al presupuesto del estado. Se realizó una análisis del impacto presupuestario con un método deterministico, según la directriz del Ministerio de Salud. Se incluyeron pacientes con probable fracaso al tratamiento de primera línea, en un horizonte temporal de 5 años para todas las tecnologías evaluadas. Se incluyeron en el análisis los medicamentos bevacizumab (uso off-label), ranibizumab y aflibercept. Las poblaciones se calcularon tanto por demanda evaluada, como por estimación epidemiológica. A modo de análisis de sensibilidad se planteó un escenario con una difusión de tecnología más lenta, un escenario con la entrada de bevacizumab y ranibizumab biosimilares en el mercado, y un escenario con la desconsideración de la inflación durante el período. El incremento del impacto presupuestario, de acuerdo con las estimativas de demanda evaluada y epidemiológica, respectivamente, fue BRL 69.493.906,95-BRL 473.226.278,78 en el caso del bevacizumab; BRL 349.319.965,60-BRL 2.378.732.103,09 en el de ranibizumab y BRL 543.867.485,47-BRL 3.703.524.490, 16 en el aflibercept. El bevacizumab se mostró la alternativa financiera más viable en todos los escenarios de estimaciones y análisis de sensibilidad. Se estimó un incremento cercano al 3\%, comparándolo con el presupuesto de 2016 (demanda evaluada). Se considera que la incorporación es viable dentro del SUS en Minas Gerais, pero sujeta a las prioridades de la gestión. La discrepancia de precios entre productos de eficacia semejante es intrigante y un tema fértil para estudios futuros.

Judicialización de la Salud; Edema Macular; Economía de la Salud; Presupuestos; Sistema Unico de Salud
Recebido em 23/Jul/2018

Versão final reapresentada em 08/Mar/2019

Aprovado em 15/Mar/2019 